$$
\text { ATO3-76SF } 71031
$$

\title{
EVALUATION OF THE MAGNITUDE AND EFFECTS OF BUNDLE DUCT INTERACTION IN FUEL ASSEMBLIES AT DEVELOPMENTAL PLANT CONDITIONS
}

$$
\text { DOE/SF/71031--T22 }
$$

D. C. Serell

S. KaPLAN

September 1980

\author{
General Electric Company \\ Advanced Reactor Systems Department \\ Sunnyvale, California
}




\section{DISCLAIMER}

This report was prepared as an account of work sponsored by an agency of the United States Government. Neither the United States Government nor any agency Thereof, nor any of their employees, makes any warranty, express or implied, or assumes any legal liability or responsibility for the accuracy, completeness, or usefulness of any information, apparatus, product, or process disclosed, or represents that its use would not infringe privately owned rights. Reference herein to any specific commercial product, process, or service by trade name, trademark, manufacturer, or otherwise does not necessarily constitute or imply its endorsement, recommendation, or favoring by the United States Government or any agency thereof. The views and opinions of authors expressed herein do not necessarily state or reflect those of the United States Government or any agency thereof. 


\section{DISCLAIMER}

Portions of this document may be illegible in electronic image products. Images are produced from the best available original document. 


$$
\text { ATO3-TLSF TIOBI }
$$

\section{EVALUATION OF THE MAGNITUDE AND EFFECTS OF BUNDLE DUCT INTERACTION IN FUEL ASSEMBLIES AT DEVELOPMENTAL PLANT CONDITIONS}

D. C. Serell

S. KAPLAN

SePtember 1980

\section{General Electric Company \\ Advanced Reactor Systems Department \\ Sunnyvale, California}

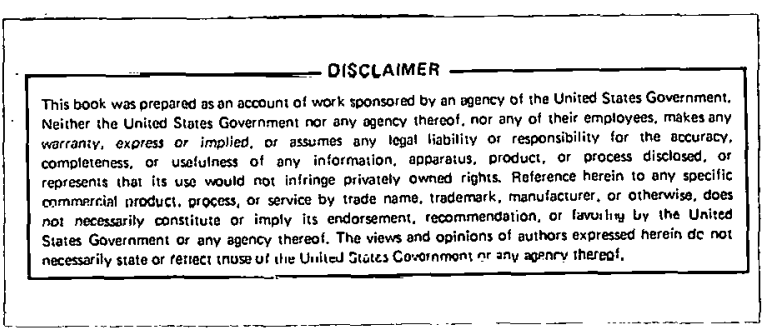

DISTRIBUTION OF THIS DOCURENT IS UMLIRITIT 


\section{CONTENTS}

\section{PÄGE}

I. Introduction and Summary , , , , , , , , 1

I1. Assembly Temperature Predictions , . . . . . . 4 Analysis Method

Fuel AsSembly Temperature UnCERTAINTy Analysis

RESULTS

III. Bundle Duct Interaction Analysis , , , , , . . 9

Method and Results

Maximum Tightness Bundle BDI Sensitivity

ANALYSES

Maximum Looseness Bundle BDI Sensitivity

AnAlyses

IV. Effects of Bundle Looseness . , , , , , , . . 14

CLADDING WEAR

Assembly local Temperature Effects

V. Effects of Bundle Tightiness , , , , , , , , , 18

Assembly Temperature Response

Temperature/Pressure Drop Feedback Effects ON BDI

VI, Summary of Results and Conclusions $, ., \cdots, \cdots, \quad, \quad, 25$ 


\section{INTRODUCTION AND SUMMARY}

The purpose of this evaluation is to estimate the magnitude and effects of irradiation and creep induced fuel bundle deformations in the developmental plant. This report does not dwell on the exact results, but will focus on the trends of the results and the ability of present models to evaluate the assembly temperatures in the presence of bundle deformation. Although this analysis focuses on the developmental plant, the conclusions are applicable to LMFBR fuel assemblies in general if they have wire spacers.

The results of evaluation of fuel assembly bundle duct interference (BDI) for the developmental plant, which uses D9 cladding and duct materials, show:

- For a 2-year fuel residence time, the maximum BDI will

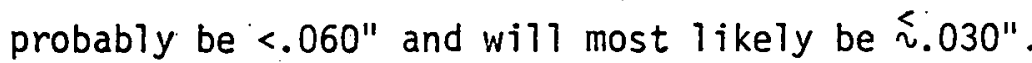

- For a 3-year fuel residence time, the maximum. BDI will probably be <0.150" and will most likely be $\approx$.120". This amount of BDI may preclude a 3-year residence time. An advanced bundle spacer design (e.g., advanced wire wrap designs or grids) may be needed.

- Sludy of the maximum "Tooseness" assembly porosity during life shows the porosity at the core midplane and below may range from $i 5$ up to 9 mils per ring, depending on material properties of 09 material, and to some extent on the operating conditions. Based on present experience, a porosity 26 mils per ring is excessive, and wear may be expected. 
Approximate analyses of the effects of BDI on fuel assembly temperatures in the developmental plant show that:

- The feedback mechanism is small between the BDI-produced assembly temperature/pressure drop/flow effects on the BDI analysis itself. Also, BDI is maximized if analyzed using uniform geometry $T-H$ conditions. This result was derived from one case using a 09 duct and $20 \% \mathrm{CW} 316$ cladding - it is not yet established as a general conclusion.

- The assembly flow reduction from relatively large amounts of BDI is not large ( $23 \%)$. The peak clad temperature increase in the center of the assembly is insignificant; however, edge pin hot spots could occur as a result of duct-edge pin contact due to edge pin bowing. There is some margin $\left(50-70^{\circ} \mathrm{F}\right)$ for edge pin hot spots before the edge pins would become more lifetime limiting than the hotter central pins. However, this conclusion cannot yet be made without more detailed study with rigorous thermal hydraulic and pin heat transfer codes such as COBRA, THT and/or FATHOM.

Regarding the ability of present methods to evaluate thermal-hydraulic/ mechanical interaction effects likely to occur in LMFBR fuel assemblies:

- The ability to predict the magnitude of BDI appears adequate, assuming the material creep-swelling properties and uncertainties are known. An important, but not exact input to this analysis is the duct temperature profile, which must come from a multi-assembly analysis. 
- Methods are needed to evaluate the detailed assembly thermal hydraulic effects of bundle deformations. Presently, the deformed geometry thermal hydraulic test data base is inadequate to verify the models. COBRA-WC or its equivalent represents a minimum level of $\mathrm{T}-\mathrm{H}$ code rigor needed to analyze bundle deformations.

- Perhaps the toughest analysis problem to solve is the thermal hydraulic and mechanical feedback problem of edge pin bowing. Bounding thermal-hydraulic analyses might provide a near-term method of circumventing this problem by simply assuming edge-pin duct contact if the resulting hot spots are acceptable and are not assembly lifetime limiting. 


\section{ASSEMBLY TEMPERATURE PREDICTIONS}

The first step in evaluating the effects of bundle deformations on assembly temperatures is to establish the base case temperatures for the assembiy of most interest. Key assembly geometric parameters of the developmental plant fuel assemblies used for this evaluation are shown in Table 1 and Figure 1 . The main differences between this design and the FFTF driver fuel design is the $D 9$ (vs. $20 \% \mathrm{CW} 316 \mathrm{SS}$ ) cladding, wire and duct material, larger pin diameter $\left(0.275^{\prime \prime}\right.$ vs. $\left.0.230^{\prime \prime}\right)$, tighter $P / D$ ratio (1.18.vs. 1.24) and number of fuel pins per fuel assembly (271.vs. 217). The fuel pin spacers are both straight-start wire wrap with a 12 " wire wrap helical pitch. There is some dissimilarity in $H / D(12 / .275=44$ vs. $12 / .230=52$ for FTR).

The CDS Phase II core-wide thermal hydraulic analys is identified the highest power and temperature fuel assembly. This anaiysis was done with the CORTEM core thermal hydraulic code, which is part of the CORTAC-3D code (1). The high power assembly is surrounded by five fuel assemblies and the highest powered internal blanket assembly. The local power distribution is virtually flat. Study of the CORTEM-calculated duct temperatures shows this high power assembly is losing a small amount of heat for batchaverage BOEC* conditions, and is gaining only a small amount of heat at EOEC*. Therefore, single assembly (adiabatic duct walls) analysis will be conservative for BOEC conditions and slightly (but insignificantiy) nonconservative at EOEC.

More detalled single assembly analysis was then performed with the fast-running MONGOOSE code. The basic structure of the code is from the

* NOTE: BOEC = Beginning of Equilibrium Cycle

EOEC = End of Equilibrium Cycle 
'PACT2 probabilistic T-H analysis code

(3) with input/output changes for convenience and with three basic modeling updates:

a) The mixing coefficient due to turbulence and wire wrap sweeping is from the Chiu (MIT) model (5);

b) The flow split between the assembly edge and interior subchannels is determined from the chtu model ${ }^{(6)}$;

c) The coolant-cladding (film) heat transfer coefficient is consistent with the recommendations of Kazimi (7) for CRBRP.

The code has convenient options for modifying these and the other inputs to make sensitivity or probabilistic analyses. MONGOOSE has the same noding and numerical analysis structure as CORTEM, i.e., an across-flats "strip model" of the assembly cross-section shown in Figure 2. This noding structure can work very well in the absence of a large power skew, in which case a "peripheral swirl flow" model is needed. Such modeling is included in more detailed thermal hydraulic codes (e.g., PNL developed codes CORTRAN, COBRA-WC), which will be used in later design analyses; however, these codes are expensive to run and do not significantly improve upon the temperature result for uniform geometry without large power skews. In spite of the lack of an inter-asselibly heat transfer model, the single assembly codes such as MONGOOSE are generally conservative for fuel (or blanket) rod temperature analysis because the high temperature assemblies are of most interest and they are losing heat to adjoining assemblies. Therefore, the single assembly analysis which does not allow the heat loss will give a slightly high, conservative fuel clad temperature result.

The initial thermal hydraulic analysis was made assuming uniform geometry. This procedure has been generally considered to yield conservative temperatures for the central rods in an assembly, $(4),(9)$ which for uniform geometry are the high temperature rods used in fuel melting 
potential or lifetime analyses. However, there is concern that hot spots can develop in the edge row pins due to thermal-hydraulic mechanical interaction effects, which occur due to irradiation induced creep-swelling effects. These hot spots could occur by rods contacting the duct due to rods bowing, as shown by the Reference 10 analysis which shows this occurring in the region above the fuel column in FTR driver fuel. Also, if the bundle-duct interference is excessive, the bundle will helically deform such that edge rods contact the duct in the fueled region. This situation will be investigated later in this report.

Fuel Assembly Temperature Uncertainty Analys is

The purpose of this section is to discuss the basis for the clad temperature uncertainty analysis. There are two basic areas of concern when making any uncertainty analysis:

a) What are the uncertainties, and how are they related?

b) How should the uncertainties be combined?

Concerning the values of uncertainties used: the "CDS Groundrule" values (2) were reviewed and determined to generally apply to the developmental plant, in the absence of significant thermal-hydraulic mechanical interaction effects that could cause local hot spots in the edge row pins.

There are two favored methods to be used in combining LMFBR thermalhydraulic uncertainties:

(1) The horizontal, semi-statistical hot channel factor (HCF) approach

(2) Monte Carlo method. 
The horizontal, semi-statistical HCF approach was used in the Reference 4 (CRBRP) analysis. This method is commonly accepted, convenient, and when used properly is appropriately accurate. This method gives accurate results for this application because the important statistical inputs are reasonably close to a normal distribution, and the process being analyzed is basically linear in the range of interest.* This method is similar to the "moments method" approach. The Monte Carlo uncertainty analys is method is a more fundamental method that is advantageous for special analyses when the input distributions are significantly different from normal distributions or the process being analyzed is significantly non-linear.

The method used for this analysis is the horizontal, semi-statistical HCF method. The first step in this method is to calculate the nominal clad temperature. Next, the "direct uncertainties" that are conservatively assumed to always occur are multiplied times each other, resulting in "t-direct" or " $t_{0 \sigma}:$ " These direct factors include clad hot-spots due to wire wrap or pellet-clad eccentricity, calculational uncertainties and inplant measurement uncertainties (e.g., core power measurement). The remaining uncertainties are then combined statistically, assuming they are normaliy distributed, and a normal distribution is derived with $t_{o \sigma}$ ( $t_{\text {direct }}$ ) as the expected value (mean). This process is illustrated graphically in Figure 3 . Note that " $\mathrm{T}_{2 \sigma}$ " is really with respect to the probability distribution about "Tou", not a true $2 \sigma$ temperature about the nominal temperature. Basically, this method follows the philosophy of not taking a "risk" on the uncertainty

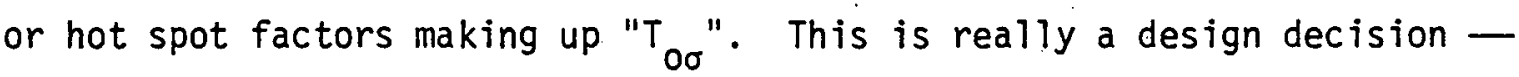
it has nothing to do with what statistical analysis technique is used. * NOTE: See Reference 4 and Reference 8 , pages $5.2-5.4$ and 6.3 for
justification. 


\section{Results}

The preliminary cladding midwall temperature results for the high power fuel assembly in the developmental plant are shown in Table 2. These temperatures are presently being used for fuel lifetime predictions. 
III. BUNDLE DUCT INTERACTION ANALYSIS

Once the fuel assembly cladding and duct temperatures are established for uniform geometry, the next step in the assembly analysis process is . to calculate the bundle duct interaction that is expected to occur during the assembly life. The highest power assembly is also the assembly that will have the most bundle duct interaction because its fast neutron fluence is also close to a maximum. In general, the higher the temperature and fluence, the more the bundle will "grow" due to swelling and creep (from fission gas pressure).

The duct will swell and creep at a different rate because of different operating temperature, pressure loading and perhaps even slightly different fluence. For some assemblies, the bundle may tighten during life, being constricted by a slower "growing" duct; for other assemblies, the opposite case may occur and the bundle will loosen.

The analysis model is a version of RODLOAD (described in Reference 11) updated to handle assembly operating condition variation during life and the properties of D9. material as well as $20 \% \mathrm{CW} 316$ SS. Primary inputs to RODLOAD are initial geometry and material properties, along with beginning and end of life fission gas pressure, assembly pressure drop, fast neutron flux and average temperature for the duct, cladding and wire wrap. The operating conditions are input as functions of elevation and time. RODLOAD calculates bundle and duct growth due to thermal expansion, swelling and creep along with bundle and duct stress. In the case of bundle-duct interference, RODLOAD calculates the average $P / D$ and clearance between the edge rods and the duct using correlations derived from ex-pile bundle compression tests.

RODLOAD anaiyses were performed for both two and three-year fuel residence time, using $D 9$ ducts and 09 cladding, which is the reference Developmental Plant design, and alternately D9 ducts and 316 cladding. 
The latter combination is being used to provide an approximate bound to the maximum BDI. At the Developmental Plant design conditions, RODLOAD analyses demonstrated that for the two-year residence, considerable looseness between the bundle and the duct could develop as a result of the relatively large duct creep-induced dilation and the relatively small amount of bundle swelling. The swelling was not significant because the two-year residence E.0.L. fast fluence does not significantly exceed the high swelling incubation fluence of D9. However, for a three-year residence, the fluence is high and considerable bundle tightness occurs. Reviewing the performance of several other assemblies at the vicinity of the peak power fuel assembly it was observed that the maximum bundle looseness occurred elsewhere than at the peak power assembly, because of a lower, bundle temperature coupled with a duct temperature comparable to that of the peak power assembly.

Figure 4 summarizes the results and provides a comparison of the BDI performance among assemblies as well as within the individual fuel assembly. BDI histories are shown at various axial locations for two assemblies, the dashed lines being for the peak power fuel assembly, and the solid lines being for a neighbor assembly which has a lower bundle temperature and only a slightly lower duct temperature. The peak power assembly produces the greatest bundle-to-duct tightness and its neighbor produces the greatest bundle-to-duct looseness.

Considering first the "loose bundle," the looseness history is shown. in the vicinity of the mid-core region (20" is midcore). Although even greater looseness occurs below mid-core, as a generalization, it may not be as problematic because of the lower temperatures. It can be seen that a maximum value of approximately 5 mils per ring looseness develops at the mid-core region and that the bundle looseness is of a very long duration. 
Considering the "tight bundle," or peak power assembly, it is first noted that considerable looseness also occurs at the mid-core region. The looseness is slightly less in magnitude and duration. It is further seen that the looseness occurs over the full lifetime. At the upper region of the 40-inch core, the bundle is initially loose, develops very slight tightness at the end of two years, and significant tightness at the end of three years. Of significance is that maximum tightness and maximum looseness occur at different axial locations, precluding a worst situation condition of maximum looseness followed by maximum tightness at the same location.

Al1 the analyses performed used nominal material properties and reactor operating conditions. The following paragraphs investigate the bundle duct interaction sensitivity to material properties and operating conditions.

\section{Maximum Tightness Bundle BDI Sensitivity Analyses}

The Figure 4 results indicate that significant bundle growth does not start until the "incubation fast fluence" is reached. For the maximum tightness bundle (which is also the high power bundle), significant growth does not start until $\sim 1.2$ years; which corresponds to the fast fluence incubation fluence $\left(i 9 \times 10^{22} \mathrm{n} / \mathrm{cm}^{2}\right.$ for 09$)$. If the incubation fluence actualiy turns out to be less as more test data becomes available, the BDI will be significantly affected. Likewise, if the swelling rate (once the incubation fluence is reached) is significantly different, the BDI will be affected. A sensitivity study was made of effects of changes in these factors, as well as a change in the creep rate. Figure $4 A$ roughly illustrates the swelling at a given temperature and shows the ranges of incubation period and swelling rate changes that were studied. The ranges of parameters chosen will probably bound the range of material properties that $D-9$ will exhibit. The results are shown in Table 3 for the maximum tightness assembly at the two elevations of maximum bundle duct interference. 
The following conclusions can be reached from the Table 3. results for the maximum tightness Dg imaterial assembiy:

- For a 2-year residence time, the maximum BDI will probably be <.060" and will most likely be $乞 .030 "$.

- For a 3-year residence time, the maximum BDI will probably be $<.150 "$ ".

- The creep and swelling changes in BDI. from the reference are roughily additive for a given residence time and elevation.

If the incubation time, $\tau$, is $\leq 7 \times 10^{22} \mathrm{n} / \mathrm{cm}^{2}$ and the creep and sweiling rates are greater than nominal, BDI will probably preclude a 3-year fuel residence time unless an advanced bundle spacer design. (e.g., advanced wire wrap designs or grids) is used.

Another parameter of interest is the fuel rod plenum pressure, which provides the driving force for creep of the fuel rod. In the preceding analyses a rather high plenum pressure ( $i 1440 \mathrm{psig}$ ) was used for end of life based on preliminary, bounding estimates for a 3-year life. Since then, more accurate plenum.pressure results from recent LIFE4 analyses are available. Therefore, some sensitivity cases were made to investigate the plenum pressure effect, the results of which are shown in Table 4. * The conclusion is that plenum pressure does not have a marked effect. "This is

* NOTE: The Table 4 results are on a lit.t.le different basis than those of Table 3 since updated values of duct pressure loading and temperatures (a little lower) were used. The data was generated at different elevations for convenience in matching the thermal hydraulic inputs. 
consistent with the Table 3 results which show creep (driven by the plenum pressure) not to be nearly as important as the effects of swelling.

Maximum Looseness Bundle BDI Sensitivity Analyses

Just as there is concern that too much bundle tightness will 1 imit bundle life (because of hot spots), there is concern that too loose a bundle may allow excessive cladding wear due to flow induced vibration. Figure 4 showed that for the maximum looseness bundle, using nominal 09 properties, the porosity can reach 50 - 60 mils at the core midplane or below during the second year of irradiation, continuing through the third year. Since 50 mils porosity translates to $5 \mathrm{mils}$ per ring and since significant wear has been observed in EBR-II fuel bundle tests with this porosity or greater, there is cause for concern.

To check the sensitivity of the maximum looseness assembly BDI to the D9 material properties, the cases shown in Table 5 were made for the 20 " (core midplane) and 24" locations above the bottom of the active fuel. The worst case combination of maximized creep and minimized swelling show i20\% increase in 1ooseness over the Figure 4 results. As was also the case for the maximum tightness bundle sensitivity cases, the fission gas pressure in these cases for the maximum looseness bundle at EOL was set high based on preliminary analyses. If an updated, lower end of life plenum pressure was used for the maximum looseness assembiy, the looseness would increase by $\ddot{0} 10 \mathrm{mils}$.

As a result of study of the maximum looseness assembly porosity during life, it is concluded that the pornsity at the midplane and below will range from is up to 9 mils per ring, depending on material properties of D9 material and to some extent on the operating conditions. Based on present experience, this porosity is excessive and wear problems may be expected. 


\section{IV: EFFECTS OF BUNDLE LOOSENESS}

Cladding Wear

As was described in the previous section, the "maximum looseness" fuel bundle in the developmental plant may have a porosity during the latter part of life that ranges from $\sim 5$ up to 9 mils per ring at the fuel midplane and below. The amount of porosity will depend to a large extent on the D9 material properties, and to some extent on the operating conditions. Since fuel pin cladding wear has been observed in EBR-II bundle tests with $\geq 5$ mils per ring porosity, there is cause for concern. This concern is heightened by: (1) recent JOYO fuel wear experience and (2) the fact that the U.S. in-pile fuel wear experience has been with the relatively short fuel pins in EBR-II, whereas longer pins would show more vibration. A compensating factor, however, is that the .275" diameter developmental plant pins wiil be stiffer than most pins tested to date, which should reduce the vibration and wear. If the lower region of the fuel is allowed to vibrate due to excessive porosity, it is possible that the entire pin would vibrate and that wear could be highest in the upper regions of the fuel pin where the contact loads are greater due to less porosity and the wear resistance is lower due to higher temperature. Present models (14) and tests do not appear to allow for axial varjations of bundle porosity nor is there test experience with porosities much larger than 7 mils per ring. Based on present experience, a porosity $\gtrsim_{6}$ is excessive, and wear problems would be expected with some of the developmental plant fuel assemblies.

Assembly Local Temperature Effects

If the bundle porosity becomes too large there is the possibility that the assembly local temperature distribution could be affected. There are three basic bundle geometric configurations that could be possible for a loose bundle: 
(1) The pins could be evenly distributed such that the gaps between wires and pins or ducts are uniform throughout the bundle;

(2). The pins could be close-packed toward the bundle center, leaving excess flow area in the peripheral region of the assembly;

(3) The bundle could be close-packed but shoved to one side of the duct, leaving a large space on one side of the bundle periphery.

Of course, these cases are idealized; an actual case could be any combination of the above. At start of life, the first case is clearly the most probably due to the fact that each fuel pin is not perfectly straight and each pin has some random bow, which causes a uniform distribution of pins within the duct due to the springy nature of the long rods. However, during operation, fuel pin or duct bowing due to thermal and irradiation swelling gradients could conceivably cause the second geometry case, or a combination of the last two. This combination case might see the bundle pushed against one side of the duct where the wire separates the pins and the duct at one elevation, with the bundle helically corkscrewing as the wire wrap rotates up the bundle. The third case could conceivably exist in a situation of significant duct bowing, leaving a gap along one side of the duct for a significant axial length. This last case could cause significant temperature skew. In pile instrumented assembly tests have shown similar bundle geometric skewing (16) and temperature skewing of Rapsodie assemblies. Temperature skewing of the EBR-II XX08 instrumented assembly $(18)$ is pulentially attributed to geometric non-uniformity. (However, the temperature skewing in the XX08 assembly appears more likely due to inlet flow maldistribution.) 
MONGOOSE thermal hydrautic studies of the first case show that the assembly temperature distribution is not affected by uniform distribution of the assembly tolerances - this is because the bundle is still uniform and the subchannel flow splits and coolant mixing are not really changed. The second case where the bundle is assumed to be closely packed together at the middle will yield higher central pin temperatures because more coolant flow will go to the already overcooled edge subchannels. This is not a very realistic case, but it provides a potential bounding case to study in the absence of understanding of how the bundle will deform. Code models may not handle this case well, however, because no test data exists to validate: the models for cases in which pin-to-pin or pin-to-duct gaps are significantly larger than the wire wrap diameter. Likewise, no test data exists for the third case mentioned where the bundle is postulated to be pushed to the same side of the duct at all elevations. This last case would result in the highest fuel pin temperatures of the three cases.

At present, assembly analysis methods are not adequate to clearly establish what pin geometry will exist in a given high porosity situation. The fuel pin bowing methods described by $D$. P. Chan in Reference 19 appear. to be a good start in understanding pin bowing mechanical behavior; however, that method does not include any thermal hydraulic feedback effects and is probably too conservative as a result. Thermal hydraulic methods are therefore needed to estimate these thermal-hydraulic-mechanical feedback effects. Such.T-H methods must initially be on the COBRA-WC (or equivalent) level of modeling detail; as experience is gained the T-H feedback effects might be parameterized into some sort of more simple, less costly T-H models.

For small amounts of bundle porosity (e.g., $\delta_{5}$ mils per ring), the bundle "springiness" arguments (15) should be sufficient to justify the uniform assumption of tolerance distribution of Case \#1, in the absence of 
significant fuel pin bowing localized effects. However, in the case of large amounts of porosity (and perhaps even for small amounts), worst-case, penalizing-type calculations would probably be required for licensing analyses (i.e., where the burden of proof is on the designer) in the absence of reasonable, mechanistic analytical methods.

The excessively loose bundle situation (i.e., i5 mils per ring) is a more difficult case to analyze than that of moderate amounts of bundle-duct tightness. This is because for the the latter case, the bundle geometry (e.g., wire-pin-duct contact points) can be reasonably well defined as a platform for doing individual pin bowing calculations, whereas, for the first case, the overall bundle geometry is not even established. 


\section{EFFECTS OF BUNDLE TIGHTNESS}

\section{Assembly Temperature Response}

As was shown in an earlier section of this report, for the maximum bundle-duct tightness fuel assemblies in the developmental plant core, the bundle-duct interference may approach $0.150^{\prime \prime}$ for a 3-year residence time. Even for the 2-year residence time case, the interference will be $.030-.060 "$. The next question is, "irnat effect will this tightness have on bundle temperatures?" This section addresses this question.

As the bundle-duct interference increases, the average pin $P / D$ will decrease because of the pin dispersion phenomenon measured in ex-pile bundle compression tests. (12) At the duct face where the wire wrap is between the edge pins and the duct, the spacing will remain equal to the wire diameter; on the duct face $180^{\circ}$ away, the spacing between the edge pins and duct will decrease. Figure 5 shows the bundle-duct minimum clearance versus across-flats duct compression in the Reference 12 bundle compression tests. These tests were made with various wire wrap configurations with the FTR fuel bundle design. For the straight-start $\left(0^{\circ}-0^{\circ}-0^{\circ}\right)$ design it is expected that the edge pins will not contact the duct before $i .120^{\prime \prime}$ bundle compression. For the $0^{\circ}-0^{\circ}-0^{\circ} /$ wireless design (55 pins w/o wires) and staggered start designs, the bundle duct contact is not expected until well over 0.200 " compression.

A study of the subchannel flow area changes for $0.100 "$ across-flats compression of the straight start FTR bundle was made, and the results are shown in Figure 6 . The largest area changes occur in the edge subchannels, as would be expected. The flow areas of the interior subchannels were not significantly affected, except for small changes near the location where the wire wrap contacts the duct. Therefore, it would be expected that 
the thermal hydraulic code models based on uniform geometry test data should work fairly well for interior subchannels. Significant development work would be needed, however, to adequately model the exterior subchannel $\mathrm{T}-\mathrm{H}$ behavior in the presence of significant ( $\left(\succsim .050^{\prime \prime}\right)$ amounts of bundle duct interference.

As a means of estimating the potential assembiy temperature effects of bundle duct interference, some MONGOOSE analy.ses were made for the maximum tightness bundle in the developmental plant. As mentioned earlier, the interior subchannel geometry is not too different from uniform geometry; therefore, the Chiu mixing coefficient model (5) in MONGOOSE should be good. The main shortcoming of MONGOOSE is the axially constant, symmetrical edge subchannel flow area assumption. For example, if the edge pins are assumed to touch the duct on one side and be spaced away from the duct on the $180^{\circ}$ face by one wire diameter, MONGOOSE wOuld assume an average spacing of $(0.0+$ wire dia. $) / 2$ between the edge pins and the duct. This would give an average temperature for the edge pins and duct, but would not indicate the local subchannel temperatures well nor would it calculate the edge rod hot spot temperature at the point of pin-duct contact. In spite of this shortcoming, the analysis should give some useful insight on the assembly temperature trends.

Cases were analyzed with varying amounts of bundle-duct interference, using rod, wire and duct dimensions taken from a RODLOAD run for the developmental plant fuel. A 2 year residence time was assumed, the duct material was $D 9$ and the clad and wire material was assumed to be $20 \% \mathrm{CW}$ 316 SS in order to get a rather large amount of bundle duct interference to magnify the temperature trends. Two different wire wrap spacer systems were assumed: the $0^{\circ}-0^{\circ}-0^{\circ} /$ wireless bundle was assumed for . $233^{\prime \prime}$ and one of the .145" BDI cases, because the bundle duct clearance w 111 be $>0$ even for this large amount of BDI. Two other cases, .076" and .145" BDI were run 
for the straight start bundle. The total flow was kept constant for all cases. The results are shown in Table 6 . As can be seen, as BDI increased, the bundle maximum coolant and clad temperature went down. This occurred for three reasons:

(1) The narrowness of the edge subchannels forces some of the flow from the overcooled edge subchannels to the central subchannels;

(2) The decreased hydraulic diameter of the subchannels increases the clad heat transfer coefficient*; and

(3) The larger pin diameter $(\sim 5 \%)$ increases the clad heat transfer area.

Another conclusion from Table 6 is that the average duct temperatures increase as BDI increases. However, the increase in average edge pin temperatures (which follow the average duct temperatures) is not large. This allows for significant margin $\left(50-70^{\circ} \mathrm{F}\right)$ for edge pin hot spots (for potential ductedge pin contact due to edge pin bowing) before the edge pins would become more lifetime limiting than the hotter central rods. However, the conclusion cannot be made that the edge pin life will not be worse without more detailed study with rigorous thermal hydraulic and pin heat transfer codes. Another cause for optimism that the edge pin temperatures will not exceed the central pin temperatures stems from the fact that the Table 6

* NOTE: The Nusselt Number, $N u=\frac{h D_{h y d}}{k}$ doesn't change much, but $h=\frac{N u K}{D_{h y d}}$ increases as $D_{\text {hyd }}$ decreases. 
values were generated assuming the bundle-duct interference was axially constant; in actuality, significant interference is expected mainly to occur in the upper portion of the bundle such that the temperature distribution at the midplane or below should not be affected. Therefore, the true average edge pin temperature rise above nominal should be about half the rise shown in the Table 6 results. 
Temperature/Pressure Drop Feedback Effects on BDI

All of the BDI analyses and sensitivity studies described in previous sections were based on uniform geometry assembly temperature and pressure drop data. Important questions are whether the BDI changes significantiy and in what direction it changes if the temperature/pressure drop effects of a given amount of BDI are fed back to the BDI analysis. This section provides an analysis of this feedback effect for the case of bundle duct interference.

Since small amounts of BDI do not significantly alter the T-H conditions, a case was set up that would result in significant $B D I$. The developmental plant maximum tightness assembly was assumed to have a $D 9$ duct with $20 \% \mathrm{CW}$ 316 SS fuel cladding and wire wrap, with a 2 year residence time. *

The first step in this analysis was to use nominal BOEC and EOEC assembly temperature and pressure drop predictions as input to the RODLOAD analysis. This analys is produced axially varying values of pin, wire and duct dimensions and the net BDI result.

Next, the average flow area at each axial section was calculated, and the change in assembly pressure drop characteristic was calculated using results from the Reference 20 compressed bundle hydraulic tests. These tests showed that compressed bundle pressure drop is best correlated by a loss coefficient per wire wrap pitch, and that this loss coefficlent ( $K_{\text {pitch }}$ ) for relatively large amounts of bundle compression is basically

\footnotetext{
* NOTE: This case is a potential fallback design if D9 fuel cladding is not acceptable for some reason. This case also is similar to an advanced alloy duct (AAD) test planned for FTR which uses $20 \% \mathrm{CW}$ 316 cladding and $D 9$ duct, with the $0^{\circ}-0^{\circ}-0^{\circ} / \mathrm{W}$ wire wrap spacer design to mitigate the effects of $B D I$.
} 
the same as $k_{\text {pitch }}$ for the uniform bundle. No matter what the bundle duct interference, the bundle pressure drop can be determined by:

$$
\Delta P_{\text {per wire }}^{\text {wrap pitch }}, \quad=\left(K_{\text {pitch }}\right) \frac{\rho V^{2} \text { average }}{2 g}
$$

Therefore, the deformed bundle axial pressure drop can be calculated using the uniform geometry $K_{p i t c h}$ and the deformed geometry average velocity squared. At this point, the revised assembly flow can be calculated assuming the total assembly $\Delta P$ is unchanged (i.e., the total core $\Delta P$ is not significantly affected by changes in the pressure drop characteristic of only one of many parallel paths). For this case, the flow will be $97 \%$ of the design flow based on uniform geometry characteristics.

Given this new flow and assuming an axially uniform BDI of $0.233^{\prime \prime}$ with .010" minimum edge-pin-duct clearance (for the $0^{\circ}-0^{\circ}-0^{\circ} /$ Wireless bundle), the I revised assembly temperatures were calculated at the end of life. These new temperatures are on the same basis as those in Table 6 for 0.233" BDI, $.010^{\prime \prime}$ clearance, except that the flow is $3 \%$ less. The change in temperatures versus the nominal EOEC temperatures at the top of the enriched fuel column are:

$\begin{array}{ll}\text { Duct average temperature: } & +22^{\circ} \mathrm{F} \\ \text { Clad average 0.D. temperature: } & +3^{\circ} \mathrm{F} \\ \text { Max. coolant temperature: } & +5^{\circ} \mathrm{F} \\ \text { Max. clad 0.0. temperature: } & +1^{\circ} \mathrm{F}\end{array}$

The final step in this analysis was to feed the revised duct average and clad average temperatures into the RODLOAD analysis to calculate the change in bundle/duct growth and BDI due to the revised temperatures and pressures. The results are shown in Table 7 . 
These results show that BDI is reduced from the previous case, meaning that the BDI calculated from uniform geometry temperatures and pressure drop is slightly larger than the temperature/pressure feedback case. The reason for the reduction in BDI is that the higher temperature and pressure loading increased the duct growth ( $\sim .002 "$ at the midplane) and the lower clad average temperatures decreased the bundle growth ( $\sim .004$ " at the midplane). This basic trend occurred for the entire length of the assembly. Also of note is that the BDI change was not very significant even for this large amount of BDI, which should have maximized the temperature/pressure feedback effects. These conclusions only strictily apply to this particular case, with $20 \% \mathrm{CW} \cdot 316 \mathrm{cladding}$ and $D 9$ duct. However, it is estimated that the conclusions may be more general due to similarities between $D 9$ and $20 \%$ CW 316 swelling and creep. For small amounts of BDI, the T-H feedback sensitivity will be much less because small amounts of BDI (e.g., ऽ.050") will not significantly affect the $\mathrm{T}-\mathrm{H}$ results. One effect that has not been considered is that of inter-assembly heat transfer. This may tend to moderate the BDI feedback effects so that they become even less significant.

Another feedback effect that should eventually be investigated is the corewide effect of the "loose" bundles. Because of looseness, the flow area is increased and $\Delta P$ decreased. Therefore, the lower $\Delta P$ "loose" bundle assemblies in the core draw flow away from the hot bundle assemblies that tend to be "tighter". This corewide feedback effect may be important and should be investigated in the core thermal hydraulic analysis for both steady state design and transient conditions. 


\section{SUMMARY OF RESULTS AND CONCLUSIONS}

The results of evaluation of fuel assembly bundle duct interference (BDI) for the developmental plant, which uses D9. cladding and duct materials, are shown in figure 4 . These and sensitivity study results of uncertainties in $D 9$ material creep-swelling properties and operating conditions show:

- For a 2-year fuel residence time, the maximum BDI will probably be <.060" and will most likely be $\approx .030^{\prime \prime}$.

- For a 3-year fuel residence time, the maximum BDI will probably be <0.150" and witl most likely be $\approx .120 "$. This amount of BDI may preclude a 3-year residence time. An advanced bundle spacer design (e.g., advanced wire wrap designs or grids) may be needed.

- Study of the maximum "looseness" assembly porosity during life shows the porosity at the core midplane and below may range from $\sim 5$ up to 9 mils per ring, depending on material properties of D9 material, and to some extent on the operating conditions. Based on present experience, a porosity $>6$ mils per ring is excessive, and wear problems would be expected.

Approximate analyses of the effects of BDI on fuel assembly temperatures in the developmental plant show that:

- The feedback mechanism is small between the BDI-produced assembly temperature/pressure drop/flow effects on the BDI analysis itself. Also, BDI is maximized if analyzed using 
uniform geometry $\mathrm{T}-\mathrm{H}$ conditions. This result was derived from one case using a D9 duct and $20 \%$ CW 316 cladding it is not yet established as a general conclusion.

- The assembly flow reduction from relatively large amounts of BDI is not large $(23 \%)$. The assembly peak clad temperature increase is insignificant in the absence of edge pin hot spots that could occur as a result of duct-edge pin contact due to edge pin bowing. Even then there appears to be some margin $\left(50-70^{\circ} \mathrm{F}\right)$ for edge pin hot spots before the edge pins would become more lifetime 1 imiting. than the hotter central pins. However, this conclusion cannot yet be made without more detailed study with rigorous thermal hydraulic and pin heat transfer codes, such as COBRA, THT, and\%or FATHOM.

Regarding the ability of present methods to evaluate thermal-hydraulic/ mechanical interaction effects likely to occur in the developmental or similar LMFBR plants:

- The ability to predict the magnitude of BDI appears to be adequate, assuming the material creep-swelling properties. are well established. An important, but not exact input to this analysis are duct temperatures, which should come from a whole core analysis. This analysis must properly model the inter-assembly heat transfer while accounting for core assembly power mismatch effects due to fuel/blanket loading history, as well as BDI effects on duct temperature and flow redistribution. 
- Methods are needed to evaluate assembly detailed thermal hydraulic effects of bundle deformations. Presently, the deformed geometry thermal hydraulic test data base is inadequate to develop or qualify the models. COBRA-WC or its equivalent represents a minimum level of $\mathrm{T}-\mathrm{H}$ code rigor needed to analyze bundle deformations.

- Perhaps the toughest analysis problem to solve is the thermal hydraulic and mechanical feedback problem of edge pin bowing. Bounding thermal-hydraulic analyses might provide a near-term method of circumventing this problem by simply assuming edge-pin duct contact if the resulting hot spots are acceptabie and are not assembly lifetime limiting. 


\section{REFERENCES}

(1) J. N. Fox and J. P. Wei, "Development and Prel iminary Verification of CORTAC-3D," GEFR-00396, September 1978 $\ldots$

(2) Letter, L. B. Levitt (AI) to Distribution, "Groundrules and Criteria Document Update for Phase II," November 21, 1979

(3) K. H. Chen, D. G. Hoover, "PACT2 - Probabilistic Analysis of Coolant and Cladding Temperatures," GEFR-00417, October 1978

(4) A. J. Friedland, "CRBRP Core Assemblies Hot Channel Factors Prel iminary Analysis," WARD-D-0050, Rev. 3, September 1979

(5) C. Chiu, W. M. Rohsenow, N. E. Todreas, "Turbulent Sweeping Flow Mixing Model for Wire Wrapped LMFBR Assemblies," C00-2245-55TR, Rev. 1, October 1978

(6) C. Chiu, et al., "Subchannel and Bundle Friction Factors and Flow Split Parameters for Laminar, Transition and Turbulent Longitudinal Flows in Wire-Wrap-Spaced Hexagonal Arrays," Appendix B of PNL-3337 by E. U. Khan, Apri1 1980

(7) M. S. Kazimi, "Heat Transfer Correlation for Analysis of CRBRP Assemblies," WARD-D-0034, Rev. 1, August 1976

(8) K. H. Chen, "Comparison of Semi-Statistical Hot Channel Factor and Monte Carlo Analyses of Fuel Assembly Coolant Temperature Uncertainties," GEFR-00354, June 1978 
(9) E. R. Schwegler, "Fuel Rod Bowing," CRBRP-ARD-0150, November 1976

(10) D. P. Chan and R. J. Jackson, "Thermal Bowing of Wire-Wrapped Fuel Pins in the Fast Test Reactor," ANS Winter Annual Meeting, San Francisco, CA, November 11-16, 1979

(11) N. A. Deane, et al., "COROPT-I, A Computer Code for. Finding Optimum LMFBR Core Designs," GEFR-00389, to be published.

(12) S. Kaplan and J. R. Punches, "The Response of Various Wire-Wrap Assemblies to Bundle-to-Duct Interaction," GEFR-00056, February 1977

(13) D. F. Naas and. E. N. Heck, "Design Fix for Vibration-Induced Wear in Fuel-Pin Bundles," ANS Trans., Vol. 24., Page 147, 1976

(14) D. P. Chan, "Preliminary Pin Bundle Design Analyses of the FC-1 Test," HEDL-TC-1659, HEDL, Richland, WA, March 1980

(15) E. H. Novendstern, "Turbulent Flow Pressure Drop Model for Fuel Rod Assemblies Utilizing a Helical Wire-Wrap Spacer System," Nuclear Engineering and Design, Vol. 22, 1972

(16) P. Blanchard, J. Leclere, H. 011 ier, "Observations on the Geometry of an Irradiated Rapsodie Subassembly," (CEN-Cadarache), ANS Transactions; Volume 23, P. 151, 152, June 14-18, 1976, Toronto, Canada

(17) D. Leteinturier and L. Cartier, "Specialists Meeting on Thermodynamics of LMFBR Fuel Sub-Assemblies Under Nominal and Non-Nominal operating Conditions,", IAEA Specialists Meeting on Thermodynamics of LMFBR Fuel Subassemblies Under Nominal and Non-Nominal Operating Conditions, Karisruhe, W. Germany, February 5-7, 1979 
(18) P. R. Betten, et al., "Predicted and Measured Temperature Profiles in a Fueled Subassembly in EBR-II," P. 963-971 of Proceedings of the Internationa? Meeting on Fast Reactor Safety. Technology, Seattle, WA, August, 1979

(19) D. P. Chan, "Thermal Bowing of Wire-Wrapped Fuel Pins in the Fast Test Reactor," (HEDL). Presented at the ANS Winter Annual Meeting, San Francisco, November 1979

(20) D. C. Sere11, "Compressed Bundle Hydraulic Test of the FTR $0^{\circ}-0^{\circ}-0^{\circ}$ and $0^{\circ}-0^{\circ}-0^{\circ}$ /Wireless Fuel Bundle Assemblies," GEFR-00515, March 1980 
TABLE 1.

DEVELOPMENTAL PLANT ASSEMBLY

DESIGN DATA FOR

THERMAL HYDRAULIC EVALUATIONS

CORE FUEL ASSEMBLY DATA

Pins per Assembly

Fuel Pin Pitch-to-Diameter Ratio

1.18

Fuel Pin Spacing Mechanisms

Straight Start,

Fuel Assembly Duct Material Wire Wrap

Duct Wall Thickness (in)

D9

Duct Outside Flat-to-Flat Dimension (in)

.140

Assembly Bundle Design Porosity (in)

5.71

Assembly Bundle Flow Area $\left(i^{2}\right)$

$\sim .024$

8.95

CORE FUEL PIN DATA

Type Fuel

Pin 0.D. (in)

$(\mathrm{Pu}-\mathrm{U}) \mathrm{O}_{2}$

Cladding Thickness (in)

.275

Pin Overall Length (in)

.0145

Cladding Material

102.

Wire Wrap Pitch (in)

D9

Wire Diameter (in)

12.

Fission Gas Plenum Location .048

Fission Gas Plenum Length (in)

Top

Bond

32.

$\mathrm{He}$ 
TABLE 2.

PEAK UNIFORM GEOMETRY FUEL ASSEMBLY TEMPERATURES

IN DEVELOPMENT PLANT (PRELIMINARY)

\begin{tabular}{|c|c|c|c|c|c|c|}
\hline \multirow{3}{*}{ Condition } & \multicolumn{6}{|c|}{ Cladding Midwa 11 Temperatures, ${ }^{\circ} \mathrm{F}$} \\
\hline & \multicolumn{3}{|c|}{ Core Midplane } & \multicolumn{3}{|c|}{$\begin{array}{l}X / L=0.9 * *, 16 " \\
\text { Above Midplane. }\end{array}$} \\
\hline & $T_{\text {nominal }}$ & $T_{0 \sigma}$ & $T_{2 \sigma}$ & $T_{\text {nominal }}$ & $T_{0 \sigma}$ & $\mathrm{T}_{2 \sigma}$ \\
\hline BOL, batch adjusted * & 930 . & 987 & 1012 & 1089 & 1159 & 1195 \\
\hline ÈOL, batch adjusted * & 886 & 924 & 942 & 1018 & 1070 & 1100 \\
\hline$-\quad-\quad-\quad-$ & - & - & - & $-\quad-$ & - & - \\
\hline BOEC, batch average * & 917 & 970 & $994^{\circ}$ & 1067 & 1133 & 1168 \\
\hline EOEC, batch average * & 895 & 935 & 954 & 1033 & 1087 & 1118 \\
\hline
\end{tabular}

* Typical top axial mid node location used for LIFE fuel pin code analysis.

* The batch adjustment factors allow for maximum potential power mismatch due to fuel loading in a 2-batch core. If an assembly has higher power at $\mathrm{BOL}$, it will have a lower power at its end of 1 ife. Batch average values do not account for this power mismatch. 
TABLE 3.

BUNDLE DUCT INTERACTION SENSITIVITIES TỌ

D9 MATERIAL PROPERTIES FOR MAX: TIGHTNESS BUNDLE

\begin{tabular}{|c|c|c|c|c|c|c|c|}
\hline Case & \multicolumn{3}{|c|}{ Uncertainties } & \multicolumn{2}{|c|}{$\begin{array}{c}\mathrm{BDI}^{* *}, 2-\mathrm{Yr}_{r} \text { Residence, } \\
\text { Inches }\end{array}$} & \multicolumn{2}{|c|}{$\begin{array}{c}\text { BDI**, } 3-Y r \text { Residence, } \\
\text { Inches }\end{array}$} \\
\hline & $\begin{array}{l}\text { Swelling } \\
\text { Factor }\end{array}$ & $\begin{array}{l}\text { Creep } \\
\text { Factor }\end{array}$ & $\begin{array}{c}\tau \\
\times 10^{-22} \\
\mathrm{n} / \mathrm{cm}^{2}\end{array}$ & $\begin{array}{l}28 \text { " from } \\
\text { Core } \\
\text { Bottom * }\end{array}$ & $\begin{array}{l}32^{\prime \prime} \text { from } \\
\text { Core } \\
\text { Bottom * }\end{array}$ & $\begin{array}{l}28^{\prime \prime} \text { from } \\
\text { Core } \\
\text { Bottom * }\end{array}$ & $\begin{array}{l}32 " \text { from } \\
\text { Core } \\
\text { Bottom * }\end{array}$ \\
\hline$\left(\begin{array}{c}0 \\
(\operatorname{Ref})\end{array}\right.$ & 1. & 1. & 9. & .007 & .010 & .080 & .095 \\
\hline 1 & 1.3 & 1. & & .020 & .019 & .112 & .121 \\
\hline 2 & 1. & 1.3 & & .002 & .011 & .078 & .103 \\
\hline 3 & 1.3 & 1.3 & & .013 & .017 & .110 & .129 \\
\hline 4 & .7 & .1 & & -.006 & 0 & .049 & .069 \\
\hline 5 & 1. & .7 & & .013 & .012 & .083 & .087 \\
\hline 6 &. .7 & .7 & $\downarrow$ & -.001 & .002 & .051 & .061 \\
\hline 7 & 1. & 1. & 7. & .029 & .032 & .101 & .119 \\
\hline 8 & 1.3 & 1. & & .048 & .046 & .139 & .152 \\
\hline 9 & 1. & 1.3 & & .023 & .032 & .099 & .127 \\
\hline 10 & 1.3 & 1.3 & & .042 & .046 & .136 & .160 \\
\hline 11 & 1. & $i$. & 5. & .050 & .055 & .121 & .142 \\
\hline
\end{tabular}

* 20" = Core Midplane El evation

** Negative is Looseness 
TABLE 4,

BUNDLE OUCT. INTERACTION SENSITIVITIES TO

FUEL ROD PLENUM PRESSURE FOR

MAX. TIGHTNESS BUNDLE (NOMINAL PROPERTIES)

\begin{tabular}{|c|c|c|c|c|c|}
\hline \multirow[b]{2}{*}{ CASE } & \multirow{2}{*}{$\begin{array}{c}\text { EOL ( } 3 \text {-Year) } \\
\text { Plenum } \\
\text { Pressure, } \\
\text { psid }\end{array}$} & \multicolumn{2}{|c|}{$\begin{array}{c}\text { BDI** After } \\
2 \text { years, inches }\end{array}$} & \multicolumn{2}{|c|}{$\begin{array}{c}\text { BDI** After } \\
3 \text { years, inches }\end{array}$} \\
\hline & & $\begin{array}{l}30 " \text { from } \\
\text { Core } \\
\text { Bottom * }\end{array}$ & $\begin{array}{l}\text { 33. } 3^{\prime \prime} \text { from } \\
\text { Core } \\
\text { Bottom * }\end{array}$ & $\begin{array}{l}30 " \text { from } \\
\text { Core } \\
\text { Bottom * }\end{array}$ & $\begin{array}{l}\text { 33. 3" from } \\
\text { Core } \\
\text { Bottom * }\end{array}$ \\
\hline 20 & $\begin{array}{l}1440 \\
\text { bounding } \\
\text { case. }\end{array}$ & .009 & .008 & .083 & .087. \\
\hline 27 & 1311 & .005 & .004 & .076 & .078 \\
\hline 22 & $\begin{array}{l}1260 \\
\text { LIFE4 output, } \\
2 \sigma \text { burnup }\end{array}$ & .004 & .002 & .073 & .075 \\
\hline & & & & & \\
\hline
\end{tabular}

** Negative is looseness

* $20^{\prime \prime}=$ Core Midplane Elevation 
TABLE 5.

BUNDLE DUCT INTERACTION SENSITIVITIES TO

D9 MATERIAL PROPERTIES FOR

MAX. LOOSENESS BUNDLE

\begin{tabular}{|c|c|c|c|c|c|}
\hline CASE & \multicolumn{3}{|c|}{ Uncertainties } & \multicolumn{2}{|c|}{ BDI**, 2-Yr Residence } \\
\hline & $\begin{array}{l}\text { Swelling } \\
\text { Factor }\end{array}$ & $\begin{array}{l}\text { Creep } \\
\text { Factor }\end{array}$ & $\begin{array}{c}x \\
\mathrm{n} / \mathrm{cm}^{2}\end{array}$ & $\begin{array}{l}20 " \text { from } \\
\text { Core } \\
\text { Bottom * }\end{array}$ & $\begin{array}{l}24 \text { " from } \\
\text { Core } \\
\text { Bottom }\end{array}$ \\
\hline$\left(\begin{array}{c}0 \\
(\operatorname{Ref})\end{array}\right.$ & 1. & 1. & 9. & -.054 & -.044 \\
\hline 1 & 1.3 & 1. & & $\therefore .051$ & -.043 \\
\hline 2 & 1. & 1.3 & & -.067 & -.053 \\
\hline 3 & 1.3 & 1.3 & & -.064 & -.051 \\
\hline 4 & .7 & 1. & & -.057 & -.046 \\
\hline 5 & 1. & .7 & & -.042 & -.036 \\
\hline 6 & .7 & .7 & 7 & -.044 & -.037 \\
\hline 7 & 1. & 1. & 7. & -.049 & -.039 \\
\hline 8 & 1. & 1. & 5. & -.044 & -.033 \\
\hline
\end{tabular}

** Negative is looseness.

* $20^{\prime \prime}=$ Core Midplane 
TABLE 6.

DUCT AND CLADDING AVERAGE TEMPERATURES WITH BUNDLE DUCT INTERFERENCE

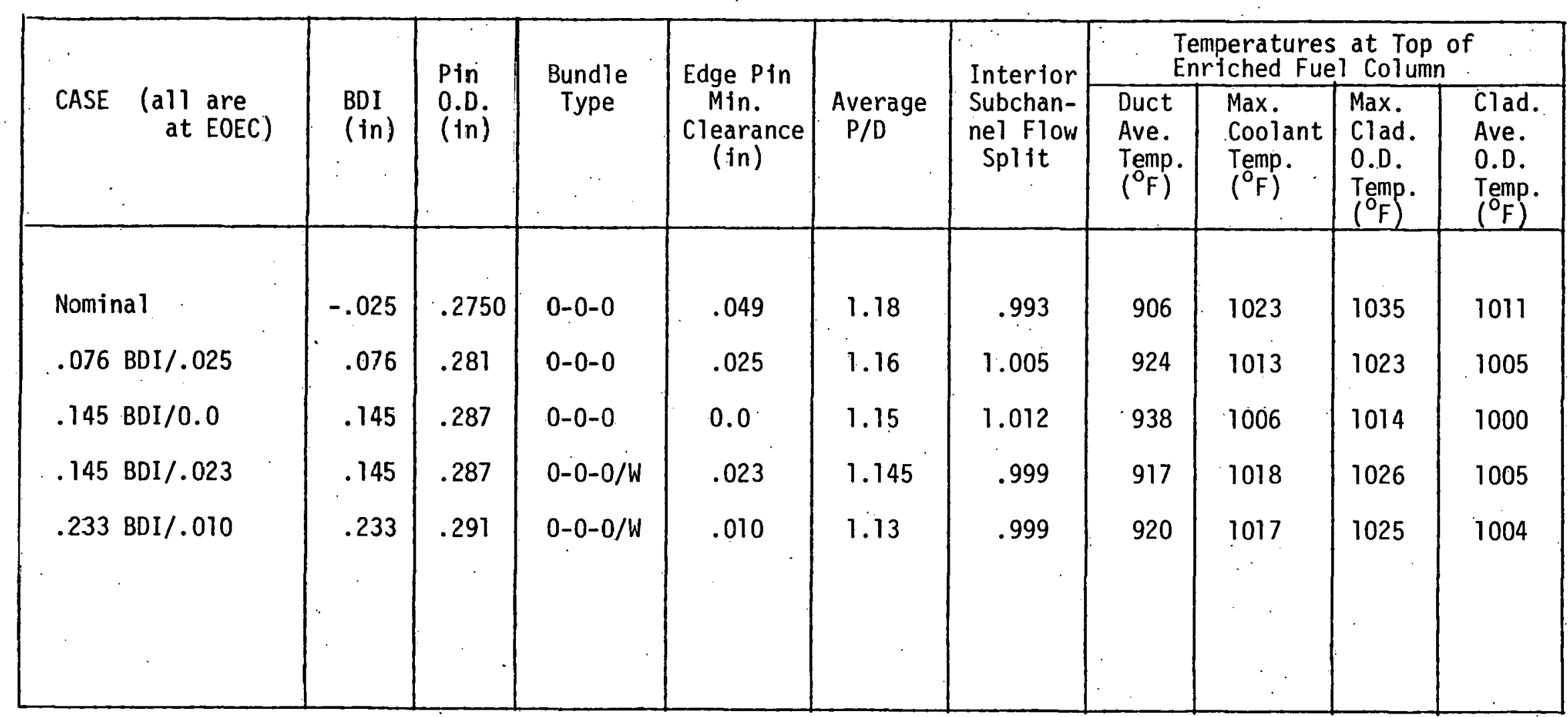


TABLE 7.

BUNDLE DUCT INTERFERENCE FOR NOMINAL T-H CONDITIONS

VERSUS THAT FOR THE T-H FEEDBACK CASE

(2-Year Residence, D-9 Duct, $20 \%$ CW 316 Cladding and Wire, Maximum Tightness Bundle)

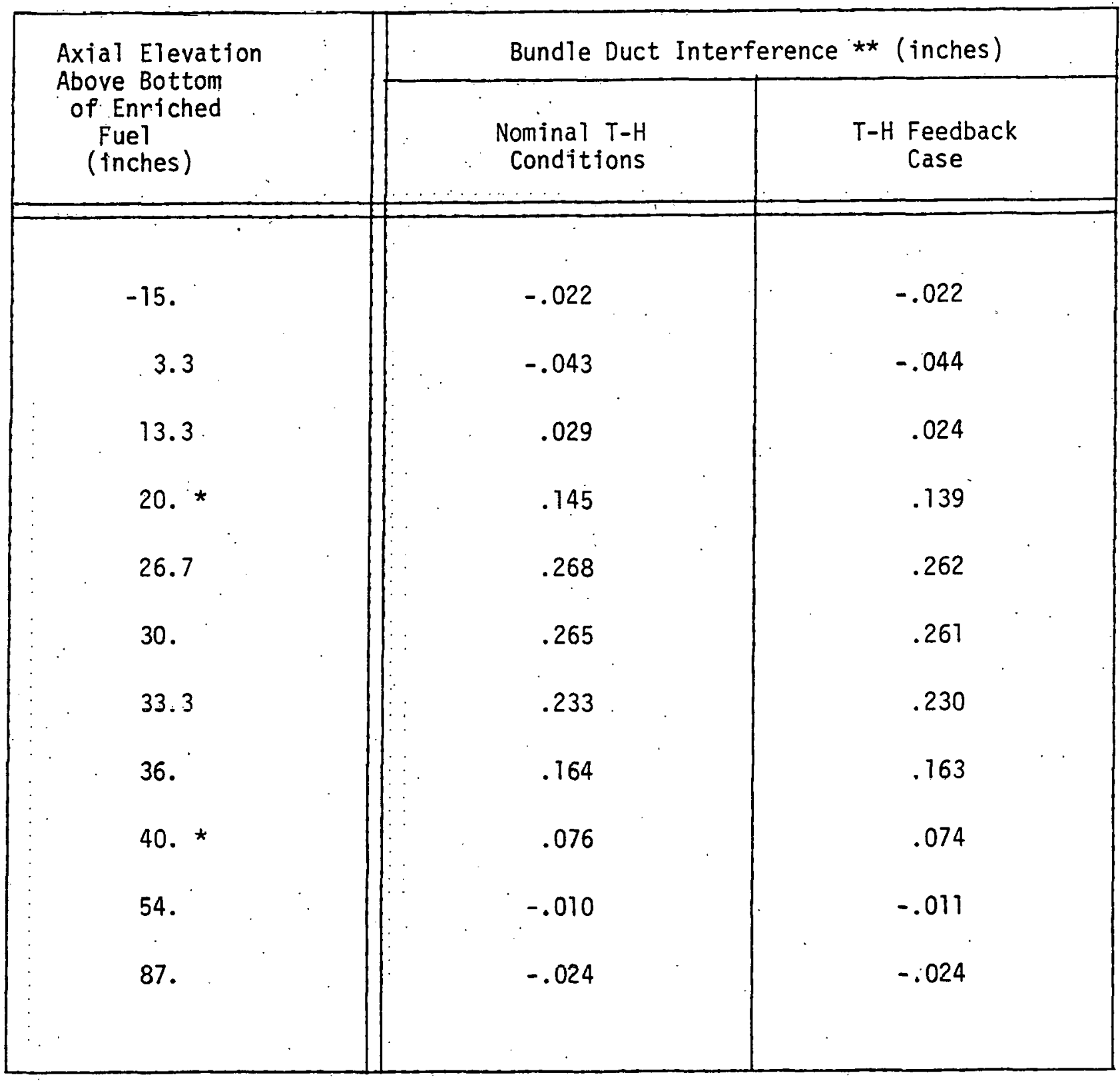

* $20^{\prime \prime}=$ Core Midplane; $40^{\prime \prime}=$ Top of Enriched Fue

** Negatfve BDI indfcates looseness 
FIGURE 1

CHARACTERISTIC LENGTHS

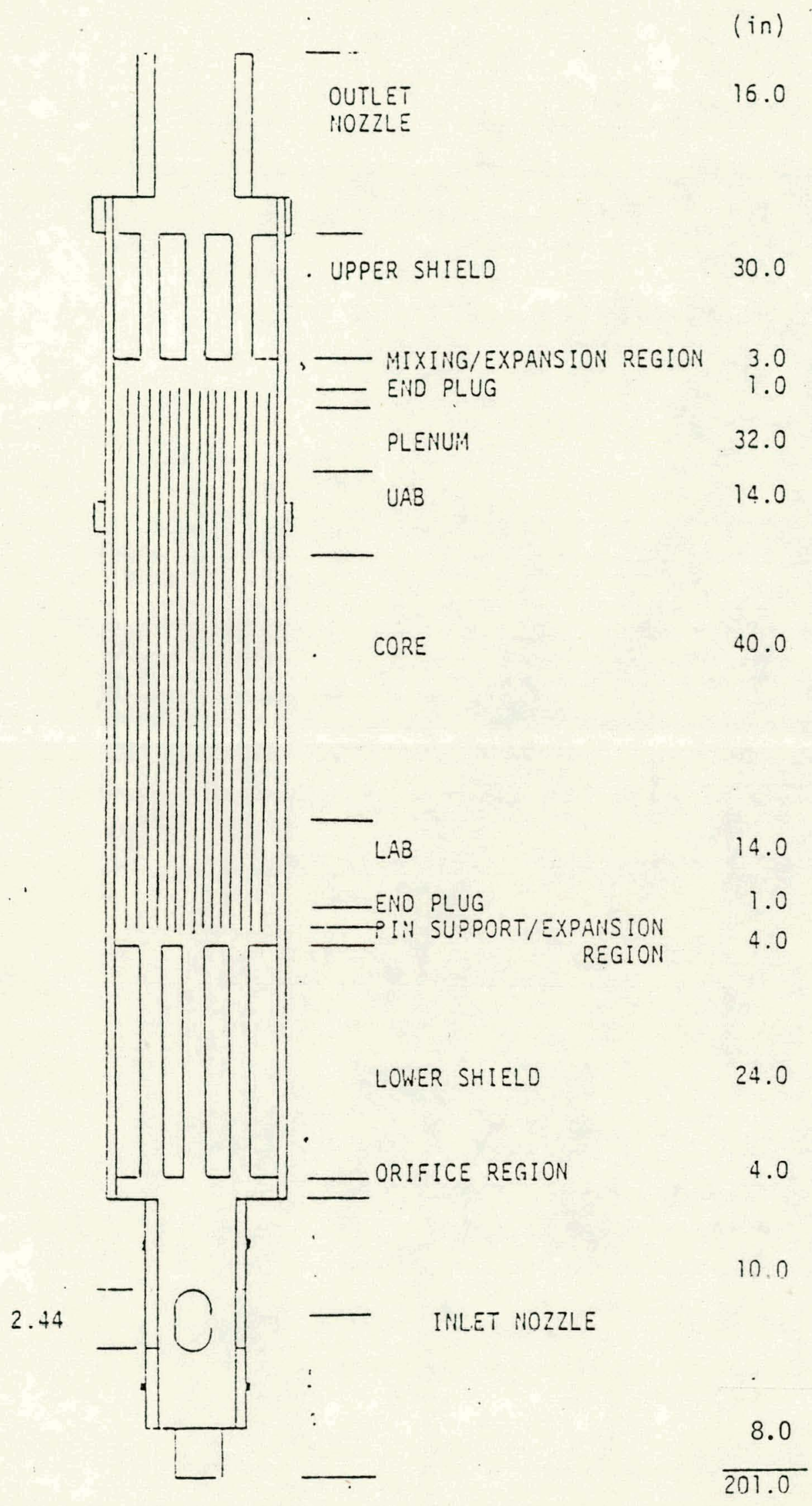



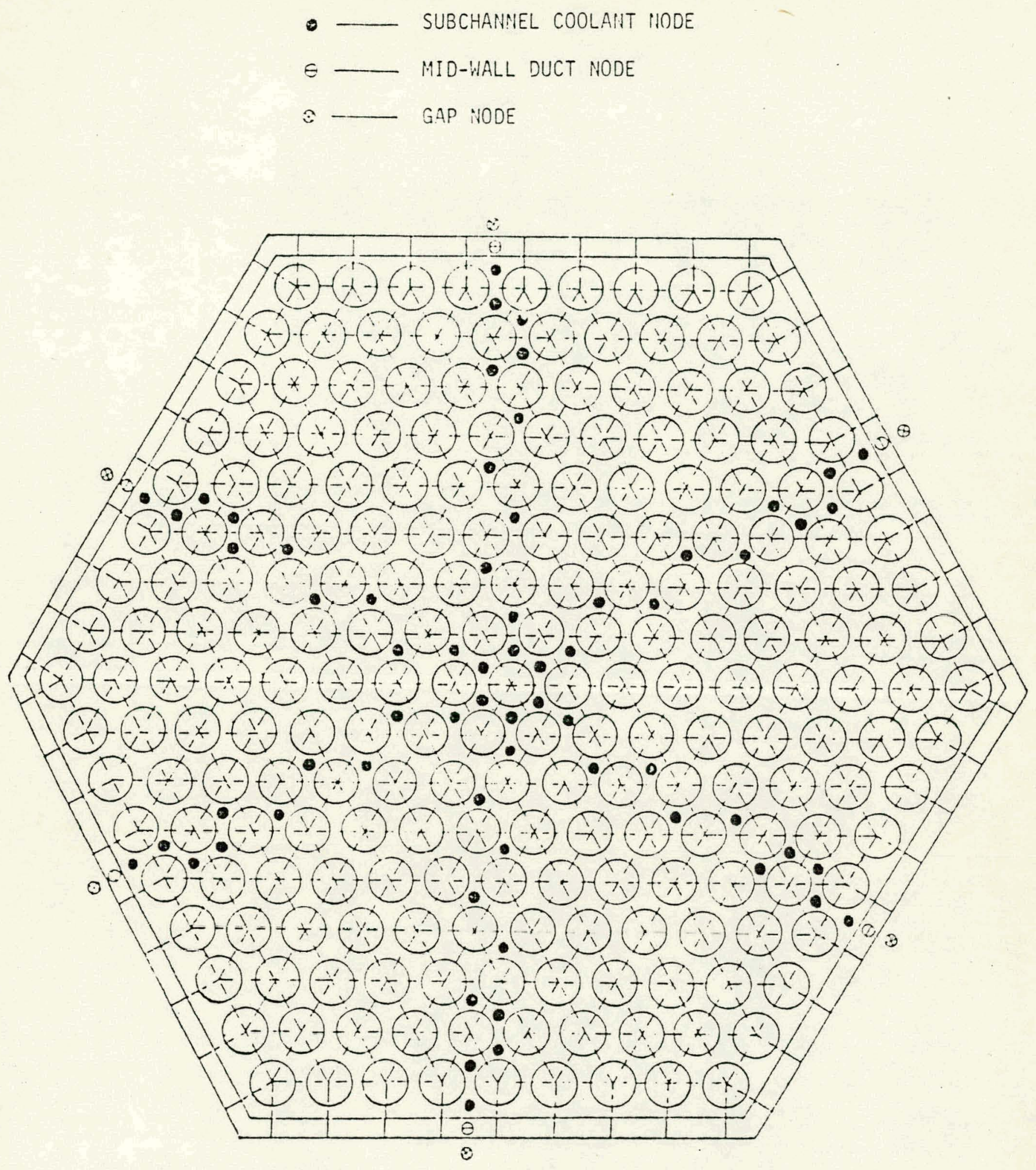

FIGURE 2. NODALIZATION FOR TYPICAL 217-PIN FUEL ASSEMBLY (CORTEM) 
i

4

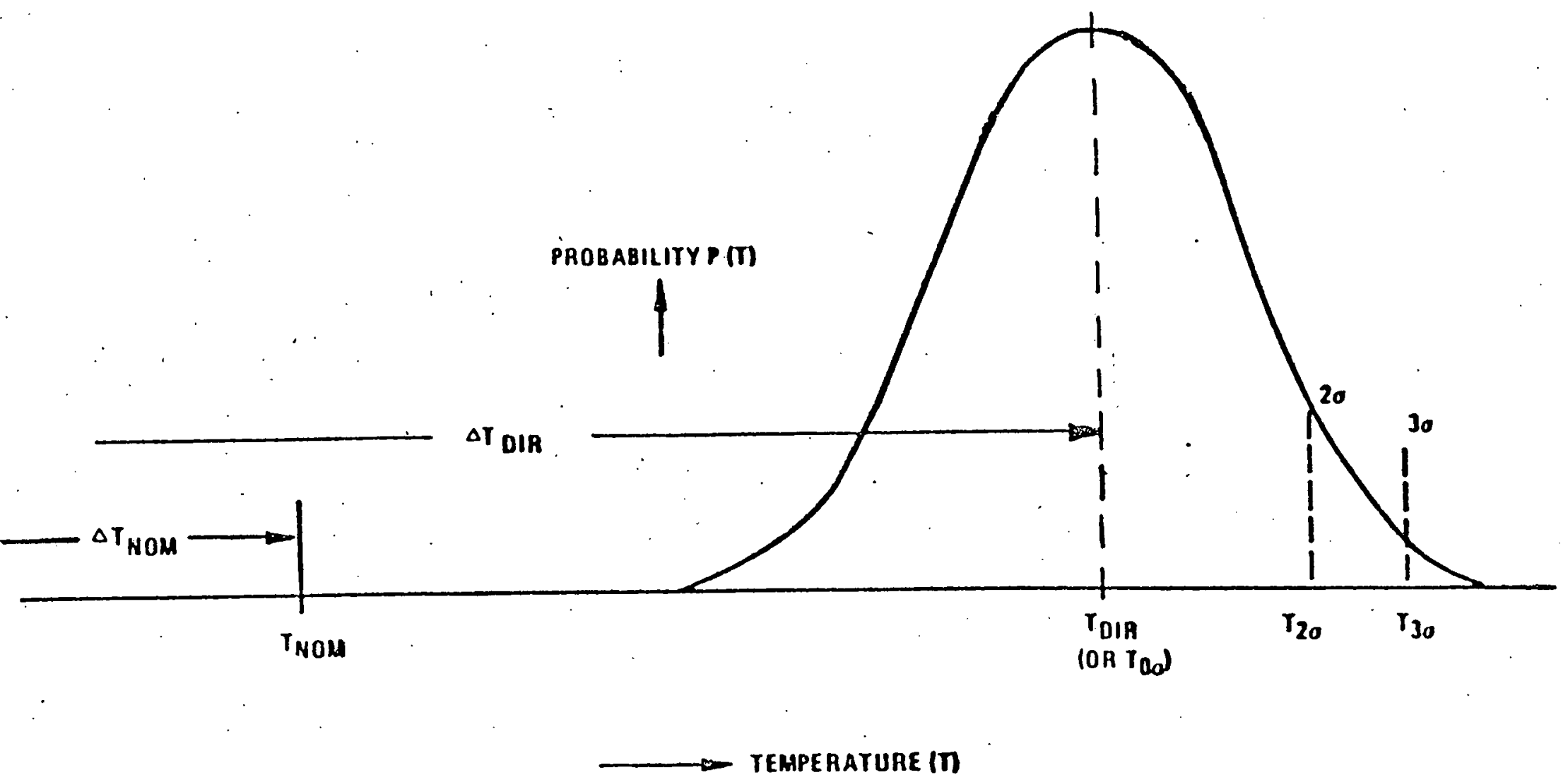

FIGUURE 3. GRAPHICAL ILLUSTRATION OF SEMISTATISTICAL METHOD 


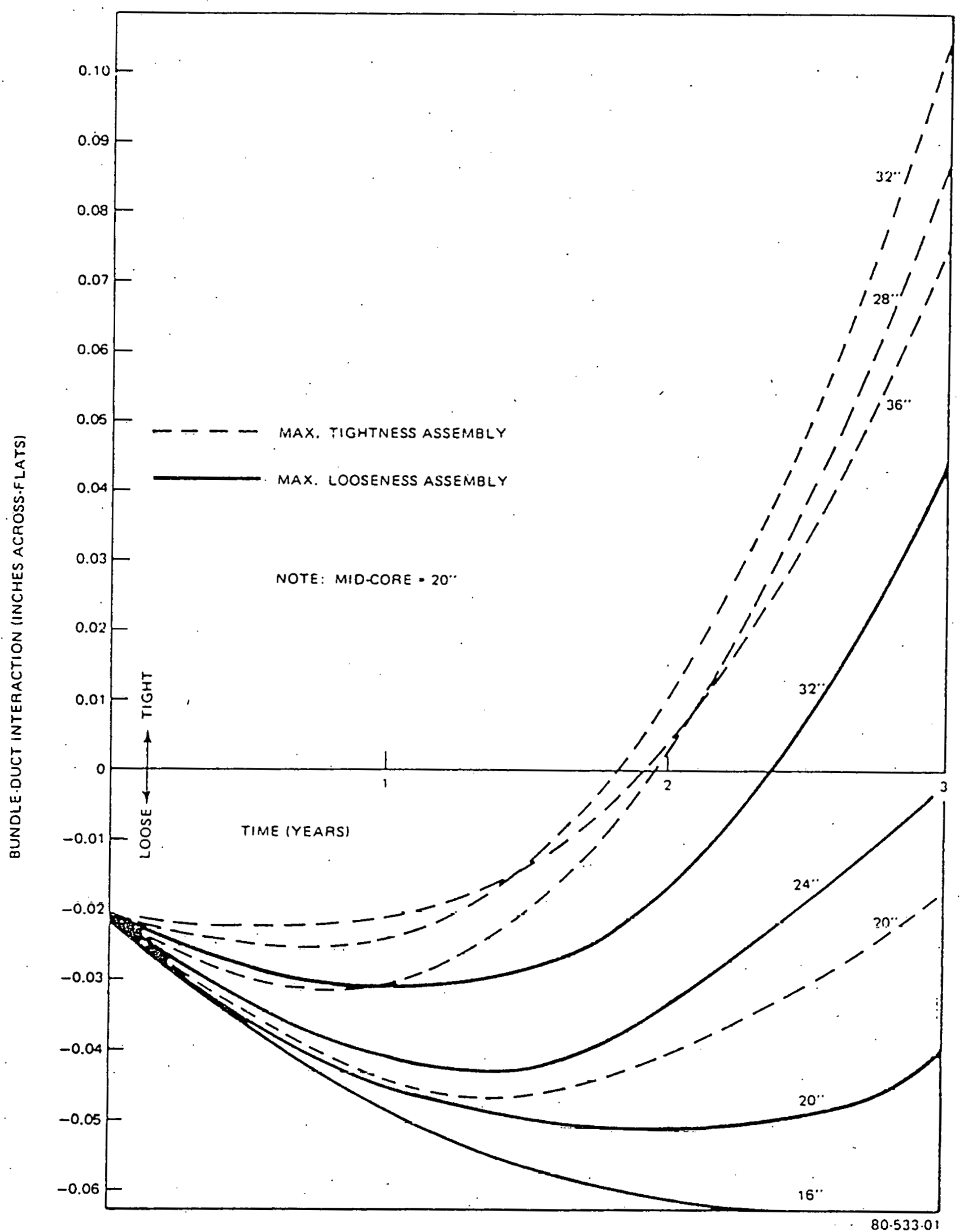

FIGURE 4. PROTOTYPE PLANT BUNDLE-DUCT INTERACTION (D9. DUICT AND D9 CLADDING) 


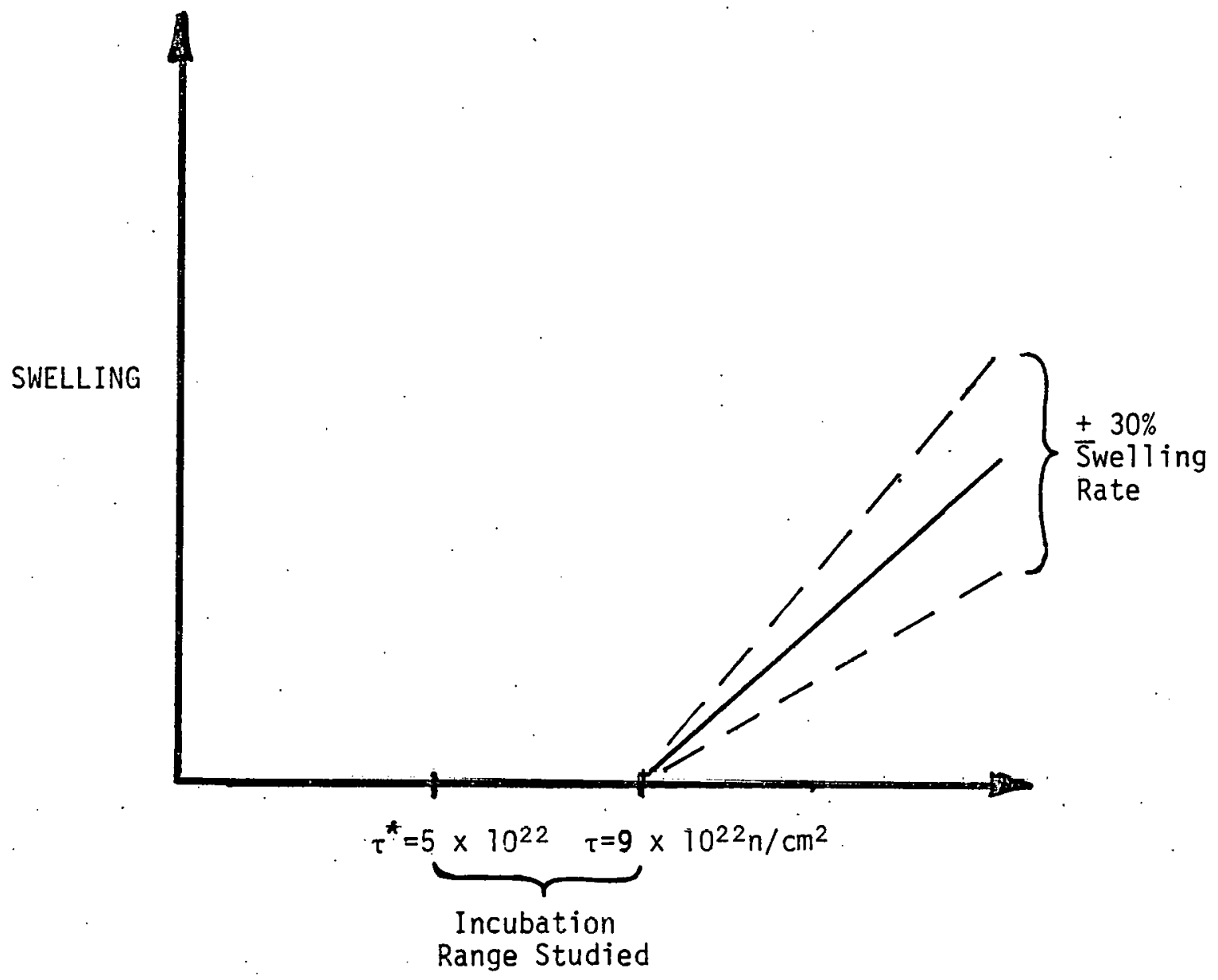

FLUENCE

FIGURE 4A. RANGE OF D9. MATERIAL PROPERTIES USED IN BDI SENSITIVITY STUDIES 


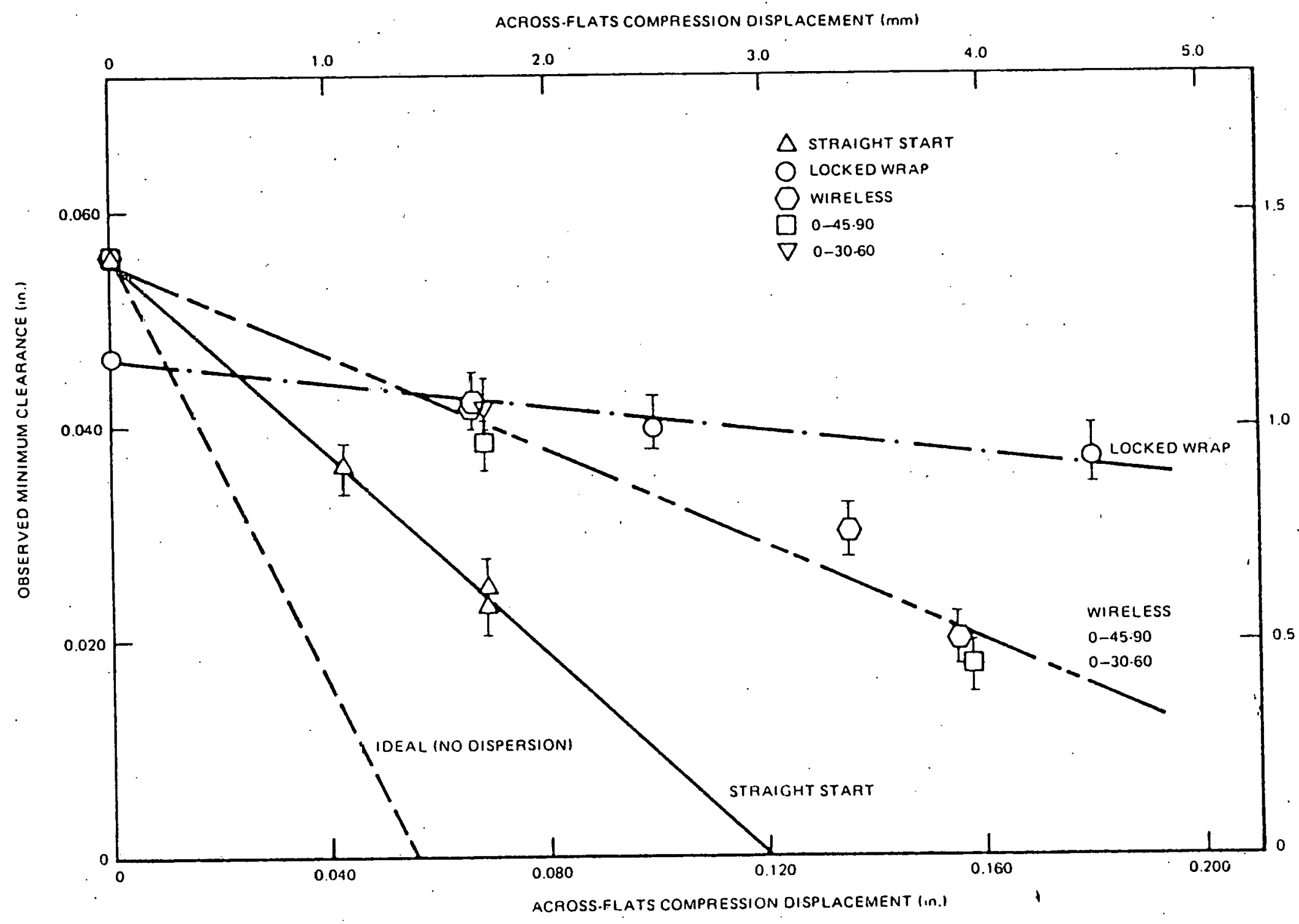

FIGURE 5. BUNDLE-DUCT OBSERVED MINIMUM CLEARANCE VERSUS ACROSS-FLATS COMPRESSION DISPLACEMENT 
Note: Average Area $11,3 \%$ Reduction $=8.0 \%$
SUBCHANNEL

AREA REDUCTION LEGEND: $\square<10 \%$

$=10-15 \%$

$15-20 \%$

$20-30 \%$

$30-40 \%$

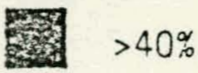
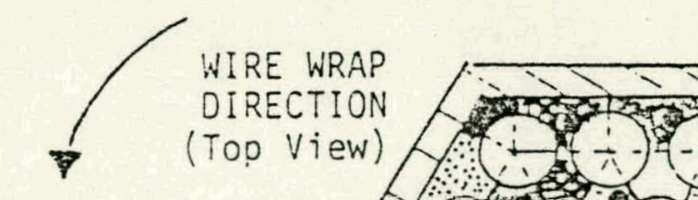

$(-+)-1+5)+1 \div \div$

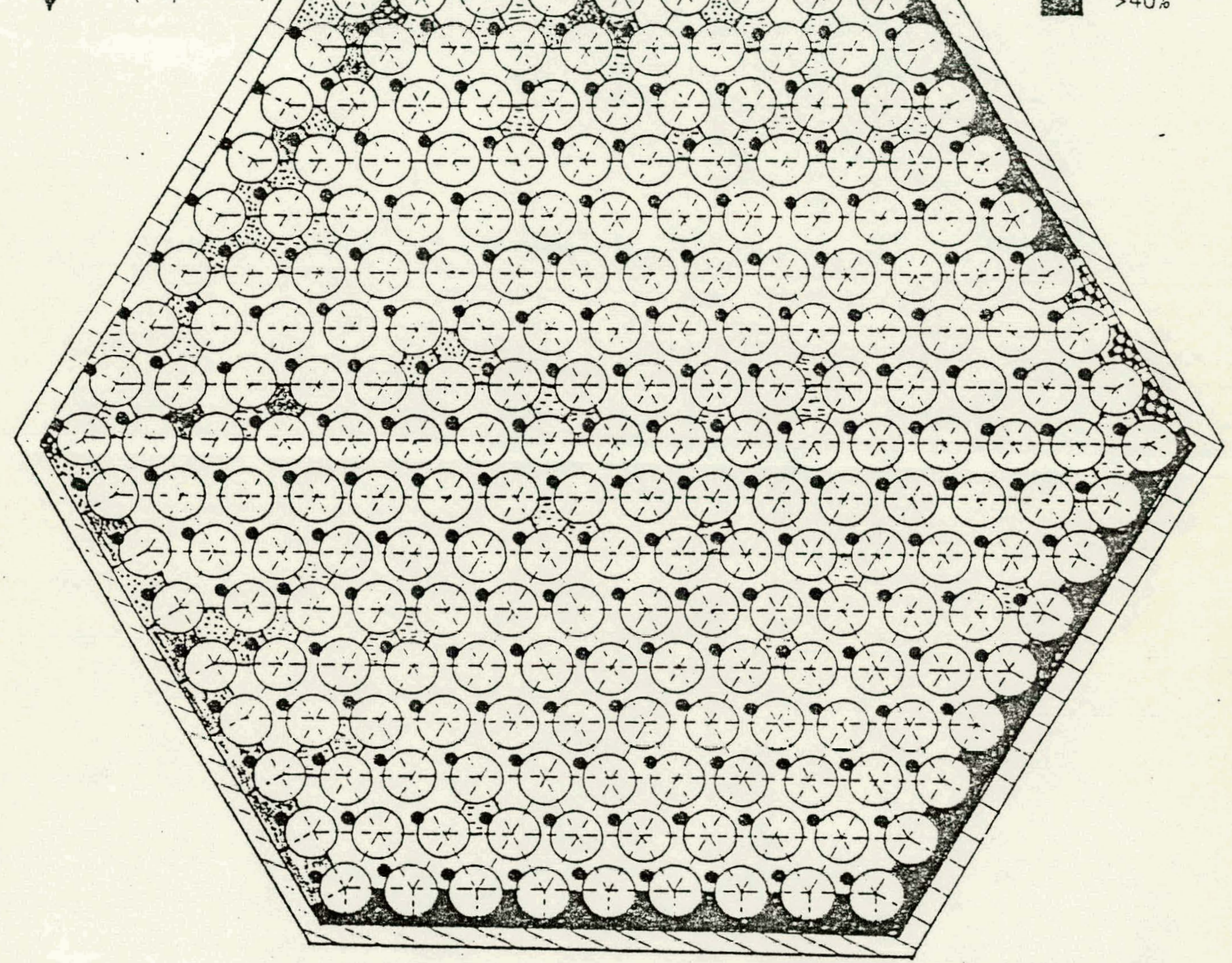

FIGURE 6.

SUBCHANNEL AREA REDUCTION OF FTR FUEL BUNDLE WITH $0.100 "$ ACROSS FLATS UNIFORM COMPRESSION 